\title{
Analytic Coarse-Mesh Finite-Difference Method Generalized for Heterogeneous Multidimensional Two-Group Diffusion Calculations
}

\author{
Nuria García-Herranz \\ Universidad Nacional de Educaćion a Distancia \\ Departamento de Ingeniería Energética, Madrid, Spain \\ and \\ Oscar Cabellos, José M. Aragonés,* and Carol Ahnert \\ Polytechnic University of Madrid, Departamento de Ingeniería Nuclear, Spain \\ Received May 28, 2002 \\ Accepted November 13, 2002
}

\begin{abstract}
In order to take into account in a more effective and accurate way the intranodal heterogeneities in coarse-mesh finite-difference (CMFD) methods, a new equivalent parameter generation methodology has been developed and tested. This methodology accounts for the dependence of the nodal homogeneized two-group cross sections and nodal coupling factors, with interface flux discontinuity (IFD) factors that account for heterogeneities on the flux-spectrum and burnup intranodal distributions as well as on neighbor effects.

The methodology has been implemented in an analytic CMFD method, rigorously obtained for homogeneous nodes with transverse leakage and generalized now for heterogeneous nodes by including IFD heterogeneity factors. When intranodal mesh node heterogeneity vanishes, the heterogeneous solution tends to the analytic homogeneous nodal solution. On the other hand, when intranodal heterogeneity increases, a high accuracy is maintained since the linear and nonlinear feedbacks on equivalent parameters have been shown to be as a very effective way of accounting for heterogeneity effects in two-group multidimensional coarse-mesh diffusion calculations.
\end{abstract}

\section{INTRODUCTION}

In order to make the solution of the few-group coarsemesh diffusion equations (hereafter called homogeneous flux solution) equivalent to that of a fine-mesh transport method (hereafter called actual flux or heterogeneous flux solution), adequate nodal equivalent parameters are required. Determining such parameters is a classical reactor physics problem, but it is even more important nowadays because of the need for improving the treatment of assembly intranodal heterogeneities in advanced three-dimensional (3-D) nodal codes. This is a key point

*E-mail: arago@din.upm.es in these kinds of codes since heterogeneity plays a very important role in determining the reactivity level and power distribution of a reactor configuration and since the recent advanced fuel designs (e.g., gadolinium in asymmetric positions) and recent core management (e.g., very long cycle duration, high fuel burnup, power uprating, low-leakage loading patterns) pose more heterogeneous and complex core conditions that result in larger interassembly and history effects.

Many nodal methods do not consider neighbor and intranodal heterogeneity effects on the equivalent parameters, and in the case of taking them into account, they decouple both problems: generating the nodal parameters and the heterogeneity treatment, accounting for them at different calculation levels. 
The purpose of this work is to present a new methodology to determine two-group equivalent parameters of application to multidimensional diffusion coarsemesh finite-difference (CMFD) calculations for heterogeneous nodes. This methodology allows one to take into account the nonlinear heterogeneous effects with its own parameters. This provides originality in contrast to most advanced nodal methods while improving the accuracy in a more computationally efficient manner.

In a previous work by the authors, ${ }^{1}$ detailed analysis of the two-group nodal homogeneized cross-section dependence on the main heterogeneity effects was presented. These effects were represented by means of local variables (neutronic and thermal hydraulic), as well as two additional generalized variables: a new spectral history index and new boundary (or neighbor) indexes. The latter are nonlinear since they are a function of their own flux solution.

By a consistent set of detailed calculations per node type [two-group pin-by-pin two-dimensional (2-D) calculations], the heterogeneity effects are isolated. It is demonstrated that correct separation of the involved effects and a suitable variable choice yield to synthesize the cross-section dependencies on the heterogeneity effects represented by both local and nonlinear variables.

The so-synthesized dependencies for each type of node are included in a library per node (or assembly) type and are used in the multidimensional coarse-mesh diffusion calculation to interpolate, node by node at the actual local conditions and locations, the two-group cross sections of the actual core configuration. Thus, identical nodes placed in different core positions will have the same nominal constants, and local, spectral history, and boundary corrections will consider the node actual surroundings, yielding different and accurate parameters for each node.

This method allows us to treat the heterogeneity effects on the cross sections explicitly, as a function of the heterogeneity variables inside the node and in its boundaries. Therefore, nonlinear two-node problems do not need to be solved during the CMFD calculation. In this way, the nodal solution is faster since the nodal parameter feedback method has shown it is a very effective way of accounting for the nonlinear effects.

In this paper heterogeneous treatment of nodal coupling factors is proposed. These nodal coupling factors complete the set of parameters that allow the homogenized diffusion equations to produce solutions that match the heterogeneous fine-mesh calculations. Through this treatment, the analytic CMFD (ACMFD) formulation recently presented by Chao, ${ }^{2}$ strictly valid for nodewiseconstant nodes, has been generalized, being now of application for actual heterogeneous nodes as well.

First, the CMFD coupling factors are discussed; then, derivation of the heterogeneous generalized ACMFD is presented. Next, an analysis of the intranodal heterogeneities is performed. Finally, the implementation in the SIMULA2D code, its application, and results are discussed.

\section{BACKGROUND: COARSE-MESH FINITE-DIFFERENCE CORRECTION FACTORS AND HETEROGENEITY EFFECTS}

Usually, the CMFD methods account for heterogeneity and coarse-mesh effects separately.

First, previous to the nodal calculation, standard assembly calculations using lattice physics codes are carried out. From these, spatially uniform cross sections in each fuel assembly type are determined, as well as heterogeneity correction factors, usually called assembly discontinuity factors ${ }^{3,4}$ (ADFs). These factors account for internal assembly heterogeneities. Logically, the boundary conditions of any node in the actual core configuration differ from the zero-net-current boundary conditions taken in the single-assembly calculations, so that the actual heterogeneity factors will differ from those. However, most of the methods take the ADFs as given for every 3-D actual core configuration. If multiassembly (colorset) calculations are performed, the heterogeneity factors estimate moreover the neighbor effects only if the actual surrounding of the node in the 3-D core has been reproduced, which is not the general case.

Then, during the standard CMFD iterative scheme, after a specified number of iterations, the so-called twonode problem calculation (a spatially decoupled calculation spanning two adjoining nodes) is performed for every interface (for all nodes and in all directions) using a higher-order advanced nodal method. These calculations update the coupling coefficients of the original problem and progressively force the CMFD values to yield the higher-order method solution. ${ }^{5}$ In other words, solving the nonlinear two-node problems allows one to include the coarse-mesh effects, that is to say the effects derived from trying to reproduce mesh-fine calculation quantities in big homogenized regions.

Finally, the methods taking into account the intranodal gradient distributions on the equivalent parameters consider these effects in the coarse-mesh calculation itself. They make a hypothesis about the flux and crosssection spatial variation inside the node. Then, cross sections are rehomogenized, and an iterative solution is performed until convergence. ${ }^{6}$

The meaning of the correction factors in the codes developed by the authors up to now is presented in the following.

\section{II.A. Correction Factors in Own Codes (Discontinuity Factors)}

The 2-D COBAYA code ${ }^{7-9}$ performs two-group pinby-pin transport-corrected diffusion calculations of 2-D whole core planes using an original local-global iteration 
method. This code includes a linear-discontinuous finitedifference diffusion procedure. The net currents per group are calculated correcting locally the finite-difference approximation of the flux gradient at the mesh interfaces by discontinuity factors. These interface correction factors imply a local modification of the actual flux at the interfaces so that a linear difference with the mesh center flux leads to the real current. Thus, for a square node (a quarter of the fuel assembly) with a side length of $h$,

$$
f_{( \pm h / 2)}^{g}=\frac{\phi_{( \pm h / 2) \text { corrected for CMFD }}^{g}}{\phi_{( \pm h / 2)}^{g}}
$$

and

$$
J_{( \pm h / 2)}^{g}=\mp \frac{2}{h} D^{g}\left\{f_{( \pm h / 2)}^{g} \cdot \phi_{( \pm h / 2)}^{g}-\bar{\phi}^{g}\right\},
$$

where all the notations are standard.

Since the local heterogeneous calculation must be performed for every assembly, the nodal approach can be viewed as an acceleration technique for solving the global heterogeneous problem. If the mesh nodes are heterogeneous, the physical meaning of these two-group discontinuity factors is taken into account as follows:

1. the internal heterogeneity effects, which modify the flux solution distributions from those obtained in homogenized regions

2. the coarse-mesh effects, since these correction factors modify locally the interface flux gradient, so that it is proportional to the current as Fick's law approximation

3. the spectral interaction effects, or the coupling effects between the fast and thermal group, since each group current is supposed to depend only on its group flux gradient.

Therefore, only one coupling coefficient by interface and group is needed to solve the CMFD nodal problem, including all the effects needed in diffusion heterogeneous CMFD calculations.

In the case of the homogeneous nodes, the discontinuity factor takes into account both the coarse-mesh and spectral interaction effects. In other words, with the COBAYA formulation, discontinuity factors are needed in calculations even for nodes with no internal heterogeneities.

Initially, we considered the possibility of including this linear-discontinuous finite-difference diffusion formulation [Eq. (2)] in a 2-D CMFD code. ${ }^{10}$ The discontinuity factors [Eq. (1)] to be used in the global reactor calculations were determined through single-assembly calculations, and their changes with the main heterogeneity effects were analyzed. Our purpose was to compute accurate discontinuity factors for the global reactor calculations, where assemblies differ from zero-netcurrent boundary conditions. In this manner, we could employ the same treatment developed for cross sections to discontinuity factors. However, as has been discussed above, the fact that such factors do involve not only effects due to the internal heterogeneity of the node but also both spectral interaction and coarse-mesh effects made impossible correct separation of their dependence with the nodal heterogeneity variables. Then, a formulation in which correction factors take only the internal heterogeneity effects into account should be considered.

Recently, Chao ${ }^{11}$ presented an ACMFD method in two energy groups for the case of homogeneous nodes, where the expressions to relate both nodal surface fluxes and currents to node-average fluxes are rigorously derived. The diffusion equation for a given node is first diagonalized in the energy spectrum space, resulting in matrix relationships involving direct dependencies with eigenvalues, eigenvectors, and energy spectra in both energy groups. In this formulation, for homogeneous node cases, it is not necessary to include ad hoc correction factors in the conventional expression of coarse-mesh finite differences in order to obtain the homogeneous solution. In other words, its own formulation includes both the coarse-mesh and spectral effects. Therefore, this formulation is adequate to be generalized for the case of heterogeneous nodes through our equivalent parameter generation methodology.

\section{GENERALIZED ANALYTIC \\ FORMULATION OF THE STATIC DIFFUSION EQUATION IN TWO ENERGY GROUPS}

A theoretical analysis of the CMFD formulation in two groups is performed while generalizing the formulation presented by Chao. ${ }^{11}$

A transverse integration procedure transforms the 2-D or 3-D partial differential diffusion equation (for each neutron energy group) into two or three, onedimensional (1-D) (ordinary) differential equations (one for each direction), coupled by the transverse leakage.

Thus, in the $x$ direction, the following matrix equation is obtained:

$$
\left[\begin{array}{c}
\nabla^{2} \phi_{f}(x) \\
\nabla^{2} \phi_{t h}(x)
\end{array}\right]-\boldsymbol{A}\left[\begin{array}{c}
\phi_{f}(x) \\
\phi_{t h}(x)
\end{array}\right]=\left[\begin{array}{c}
\frac{L_{f y}(x)}{D_{f}} \\
\frac{L_{t h y}(x)}{D_{t h}}
\end{array}\right],
$$

where the $f$ and th indexes refer to the fast and thermal groups, respectively, and the rest of the notations are standard, where matrix $\boldsymbol{A}$ is the following:

$$
\boldsymbol{A}=\left[\begin{array}{cc}
\frac{\Sigma_{a f}+\Sigma_{s f \rightarrow t h}-k_{e f f}^{-1} \cdot \nu \Sigma_{f f}}{D_{f}} & \frac{-\nu \Sigma_{f t h}}{k_{e f f} D_{f}} \\
\frac{\Sigma_{s f \rightarrow t h}}{D_{t h}} & \frac{-\Sigma_{a t h}}{D_{t h}}
\end{array}\right] .
$$




\section{III.A. Case of Homogeneous Nodes Without Transverse Leakage}

Let us consider a homogeneous region, without transverse leakage (this would correspond to a 1-D homogeneous calculation). The matrix equation to solve would be

$$
\nabla^{2} \boldsymbol{\phi}(x)-\boldsymbol{A} \boldsymbol{\phi}(x)=\mathbf{0} .
$$

If we define $-B_{1}^{2}$ and $-B_{2}^{2}$ as the eigenvalues of matrix $\boldsymbol{A}$, and matrix $\boldsymbol{R}^{-1}$ as the $2 \times 2$ matrix with columns of the corresponding eigenvectors:

$$
\boldsymbol{R}^{-1}=\left[\begin{array}{cc}
1 & r_{2} \\
r_{1} & 1
\end{array}\right]=\left[\begin{array}{cc}
1 & \frac{\Sigma_{a t h}+D_{t h} B_{2}^{2}}{\Sigma_{s, f \rightarrow t h}} \\
\frac{\Sigma_{s, f \rightarrow t h}}{\Sigma_{a t h}+D_{t h} B_{1}^{2}} & 1
\end{array}\right],
$$

we can introduce a new unknown $\psi$ by the relation

$$
\left[\begin{array}{l}
\psi_{1} \\
\psi_{2}
\end{array}\right]=\boldsymbol{R}\left[\begin{array}{l}
\phi_{f} \\
\phi_{t h}
\end{array}\right]
$$

so that the 1-D diffusion equation (5) in the energetic space can be decoupled into two equations in the modal space as follows:

$$
\nabla^{2} \psi_{m}+B_{m}^{2} \psi_{m}=0 \quad m=1,2,
$$

where the index $m$ refers to the fundamental and transient modes, respectively $(m=1,2)$. The general solution of any decoupled equation (8) is

$$
\psi_{m}(x)=E_{m} s\left(k_{m} x\right)+F_{m} c\left(k_{m} x\right) \quad m=1,2,
$$

and for Cartesian 1-D geometry,

$$
\begin{aligned}
k_{m}=\sqrt{B_{m}^{2}} \quad s\left(k_{m} x\right) & =\left\{\begin{array}{lll}
\sinh k_{m} x & \text { si } & B_{m}^{2}<0 \\
\sin k_{m} x & \text { si } & B_{m}^{2}>0
\end{array}\right. \\
c\left(k_{m} x\right) & =\left\{\begin{array}{lll}
\cosh k_{m} x & \text { si } & B_{m}^{2}<0 \\
\cos k_{m} x & \text { si } & B_{m}^{2}>0
\end{array}\right. \\
t\left(k_{m} x\right) & =\left\{\begin{array}{lll}
\tanh k_{m} x & \text { si } & B_{m}^{2}<0 \\
\tan k_{m} x & \text { si } & B_{m}^{2}>0
\end{array},\right.
\end{aligned}
$$

where the eigenvalue of the transient mode is always negative $\left(B_{2}^{2}<0\right)$.
Once the eigenvectors are obtained, the neutron fluxes become

$$
\begin{aligned}
{\left[\begin{array}{l}
\phi_{f t}(x) \\
\phi_{t h}(x)
\end{array}\right]=R^{-1}\left[\begin{array}{l}
\psi_{1} \\
\psi_{2}
\end{array}\right] } \\
=R^{-1}\left\{\left[\begin{array}{cc}
s\left(k_{1} x\right) & 0 \\
0 & s\left(k_{2} x\right)
\end{array}\right]\left[\begin{array}{l}
E_{1} \\
E_{2}
\end{array}\right]\right. \\
\left.+\left[\begin{array}{cc}
c\left(k_{1} x\right) & 0 \\
0 & c\left(k_{2} x\right)
\end{array}\right]\left[\begin{array}{l}
F_{1} \\
F_{2}
\end{array}\right]\right\} .
\end{aligned}
$$

This matrix equation contains two coefficients per node and mode, summing up a total of four nodal constants, which are to be determined by the same number of constraints. First, we choose the node-average flux in both energy groups and then the interface net currents per group in any of both nodal interfaces.

Expressing the nodal constants in terms of these constraints yields the following matrix relationships to the left $(-h / 2)$ and to the right $(+h / 2)$ of the node:

$$
\begin{aligned}
\boldsymbol{R}\left[\begin{array}{c}
\phi_{f t}( \pm h / 2) \\
\phi_{t h}( \pm h / 2)
\end{array}\right] & {\left[\begin{array}{cc}
\frac{k_{1} h}{s\left(k_{1} h\right)} & 0 \\
0 & \frac{k_{2} h}{s\left(k_{2} h\right)}
\end{array}\right] \boldsymbol{R}\left[\begin{array}{c}
\bar{\phi}_{f t} \\
\bar{\phi}_{t h}
\end{array}\right] } \\
= & \frac{h}{2}\left[\begin{array}{cc}
\frac{t\left(k_{1} h / 2\right)}{k_{1} h / 2} & 0 \\
0 & \frac{t\left(k_{2} h / 2\right)}{k_{2} h / 2}
\end{array}\right] \boldsymbol{R} \boldsymbol{D}^{-1}\left[\begin{array}{c}
J_{f t}( \pm h / 2) \\
J_{t h}( \pm h / 2)
\end{array}\right] .
\end{aligned}
$$

Defining as $\boldsymbol{C}^{\boldsymbol{f}}$ the first matrix and $\boldsymbol{C}^{\boldsymbol{j}}$ the second one, the previous expression results in

$$
\boldsymbol{R} \boldsymbol{\phi}_{( \pm h / 2)}=\boldsymbol{C}^{\boldsymbol{f}} \boldsymbol{R} \overline{\boldsymbol{\phi}} \mp \frac{h}{2} \boldsymbol{C}^{\boldsymbol{j}} \boldsymbol{R} \boldsymbol{D}^{-1} \boldsymbol{J}_{( \pm h / 2)} .
$$

In other words, this is a matrix equation involving four scalar equations, two per interface. From Eq. (13) it can be deduced that the fast and thermal net currents at the interfaces of the homogeneous nodes depend on the average node fluxes and the interface fluxes of both energy groups through "response matrices" that depend on the nodal eigenvalues and eigenvectors and the node size. Such matrices are node dependent, being sensitive to changes in core reactivity and material properties but being independent of the considered nodal interface. Therefore, $\boldsymbol{C}^{\boldsymbol{f}}$ and $\boldsymbol{C}^{\boldsymbol{j}}$ are the same on all the interfaces of the same node for this case.

This procedure, leading to an equation relating both nodal interface fluxes and currents to node-average fluxes, 
is of application to 1-D calculations with homogeneous nodes. For this case, it provides the ACMFD, a selfcontained analytic method in two energy groups.

In terms of net currents, Eq. (13) becomes

$$
\boldsymbol{J}_{( \pm h / 2)}=\mp \frac{2}{h} \boldsymbol{D} \boldsymbol{R}^{-1} \boldsymbol{C}^{\boldsymbol{j}^{-1}}\left\{\boldsymbol{R} \boldsymbol{\phi}_{( \pm h / 2)}-\boldsymbol{C}^{\boldsymbol{f}} \boldsymbol{R} \overline{\boldsymbol{\phi}}\right\},
$$

or in modal space

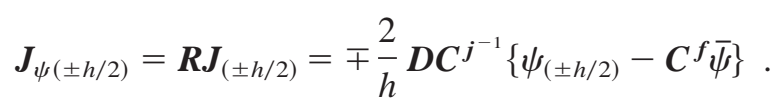

If one compares the coupling Eq. (2) in the COBAYA code and Eq. (15) in modal space, they look similar. However, the latter leads to the nodal coupling equation without the need of including any external synthetic factor in the conventional expression of CMFD in order to obtain the nodal homogeneous solution. Our previous formulation contains both coarse-mesh and spectral interaction effects, while this analytic formulation is self-contained and can be used as stand-alone without using any high-order solution over two adjacent nodes to obtain the coupling factors in CMFD. It leads to a nonlinear iterative solution because the eigenfunctions and eigenvalues in the $\boldsymbol{C}$ s and $\boldsymbol{R}$ depend on the core eigenvalue $k_{\text {eff }}$.

\section{III.B. Case of Homogeneous Nodes with Transverse Leakage}

Consider the multidimensional case of homogeneous nodes with transverse leakage. The solution to the transverse-integrated Eq. (3) is sought by combining the solution of the 1-D homogeneous Eq. (11), $\boldsymbol{\phi}_{1 D}(x)$, and the particular solutions $\boldsymbol{P}(x)$.

We have to determine a vector function $\boldsymbol{P}(x)$ in the energy spectrum space satisfying

$$
\nabla^{2} \boldsymbol{P}(x)-\boldsymbol{A P}(x)=\boldsymbol{D}^{-1} \boldsymbol{L}(x),
$$

or equivalently, we have to determine a vector function in the modal space $\boldsymbol{Q}(x)=\boldsymbol{R} \boldsymbol{P}(x)$ :

$$
\nabla^{2} \boldsymbol{Q}(x)+\boldsymbol{B}^{2} \boldsymbol{Q}(x)=\boldsymbol{R} \boldsymbol{D}^{-1} \boldsymbol{L}(x),
$$

with the general flux solution:

$$
\boldsymbol{\phi}(x)=\boldsymbol{\phi}_{1 \boldsymbol{D}}(x)+\boldsymbol{P}(x) .
$$

As in the previous case without transverse leakage, the average fluxes are related to the interface fluxes and currents as follows:

$$
\begin{aligned}
\boldsymbol{R} \boldsymbol{\phi}_{( \pm h / 2)}= & \boldsymbol{C}^{\boldsymbol{f}} \boldsymbol{R} \bar{\phi} \mp \frac{h}{2} \boldsymbol{C}^{\boldsymbol{j}} \boldsymbol{R} \boldsymbol{D}^{-1} \boldsymbol{J}_{( \pm h / 2)} \\
& -\boldsymbol{C}^{\boldsymbol{f}} \overline{\boldsymbol{Q}}+\boldsymbol{Q}_{( \pm h / 2)} \mp \frac{h}{2} \boldsymbol{C}^{\boldsymbol{j}} \boldsymbol{Q}_{( \pm h / 2)}^{\prime} .
\end{aligned}
$$

introducing the term

$$
\boldsymbol{T}_{( \pm \boldsymbol{h} / 2)}=\boldsymbol{C}^{f} \overline{\boldsymbol{Q}}-\boldsymbol{Q}_{( \pm \boldsymbol{h} / 2)} \pm \frac{h}{2} \boldsymbol{C}^{\boldsymbol{j}} \boldsymbol{Q}_{( \pm \boldsymbol{h} / 2)}^{\prime},
$$

we find the following nodal coupling relation for multidimensional homogeneous calculations:

$$
\boldsymbol{R} \boldsymbol{\phi}_{( \pm h / 2)}=\boldsymbol{C}^{\boldsymbol{f}} \boldsymbol{R} \overline{\boldsymbol{\phi}} \mp \frac{h}{2} \boldsymbol{C}^{j} \boldsymbol{R} \boldsymbol{D}^{-1} \boldsymbol{J}_{( \pm h / 2)}-\boldsymbol{T}_{( \pm h / 2)} .
$$

Note that to calculate the term $\boldsymbol{T}$ leading to the homogeneous flux solution, by Eqs. (20) and (17), the transverse leakage profile $\boldsymbol{L}(x)$ has to be known. After analyzing lower- and higher-order profiles, we have confirmed that a quadratic polynomial fit of transverse currents is quite good.

Up to this point, homogeneity assumption has been made. In the following, we show how the formulation represented by Eq. (21) can be generalized for the case of heterogeneous nodes.

\section{III.C. Case of Heterogeneous Nodes}

Consider a single-assembly calculation, with four nodes per assembly, and let us suppose it to be critical $\left(k_{\text {eff }}=k_{\infty}=1\right)$. In any given node, the interface currents are zero, $\boldsymbol{J}=\boldsymbol{0}$ :

$$
-\Sigma_{s f \rightarrow t h} \bar{\phi}_{f}+\Sigma_{a t h} \bar{\phi}_{t h}=0 .
$$

The fundamental eigenvalue is zero, $B_{1}^{2}=0$, so that the element $r_{1}$ of the matrix $\boldsymbol{R}^{-1}$ will be

$$
r_{1}=\frac{\Sigma_{s, f \rightarrow t h}}{\Sigma_{a t h}}=\frac{\bar{\phi}_{t h}}{\bar{\phi}_{f}} \Rightarrow-r_{1} \bar{\phi}_{f}+\bar{\phi}_{t h}=0 .
$$

Then, substituting Eq. (23) into Eq. (7), the function $\bar{\psi}$ is

$$
\bar{\psi}=\frac{1}{1-r_{1} r_{2}}\left[\begin{array}{cc}
1 & -r_{2} \\
-r_{1} & 1
\end{array}\right]\left[\begin{array}{c}
\bar{\phi}_{f} \\
\bar{\phi}_{t h}
\end{array}\right]=\left[\begin{array}{c}
\bar{\psi}_{1} \\
0
\end{array}\right] .
$$

So, $\bar{\psi}_{2}=0$ in any given node from the critical singleassembly calculation.

On the other hand, since there is not transverse leakage, the function $\bar{\psi}$ must satisfy the analytic formulation of Eq. (21):

$$
\left[\begin{array}{l}
\psi_{1}\left( \pm \frac{h}{2}\right) \\
\psi_{2}\left( \pm \frac{h}{2}\right)
\end{array}\right]=\left[\begin{array}{cc}
C_{11}^{f} & 0 \\
0 & C_{22}^{f}
\end{array}\right]\left[\begin{array}{l}
\bar{\psi}_{1} \\
\bar{\psi}_{2}
\end{array}\right] .
$$

Let us suppose the fuel assembly is homogeneous. Since it is a single-assembly calculation, the flux distribution profile is flat, so interface fluxes are equal to average fluxes: 


$$
\phi_{f}\left( \pm \frac{h}{2}\right)=\bar{\phi}_{f} \quad \text { and } \quad \phi_{t h}\left( \pm \frac{h}{2}\right)=\bar{\phi}_{t h} .
$$

Therefore, if Eq. (23) is satisfied, it will be also verified that

$$
-r_{1} \phi_{f}\left( \pm \frac{h}{2}\right)+\phi_{t h}\left( \pm \frac{h}{2}\right)=0 \Rightarrow \psi_{2}\left( \pm \frac{h}{2}\right)=0 .
$$

Since $\psi_{2}( \pm h / 2)=0$ and $\bar{\psi}_{2}=0$, we see that the analytic formulation is verified for this particular case of homogeneous nodes. That is, the actual fluxes in the interfaces are equal to those reproduced from coarse-mesh diffusion calculations.

Let us suppose that the fuel assembly is heterogeneous. In this case, $\bar{\psi}_{2}=0$ is still valid, but now the flux distribution profile is not flat, and therefore, at the nodal interfaces

$$
-r_{1} \phi_{f}\left( \pm \frac{h}{2}\right)+\phi_{t h}\left( \pm \frac{h}{2}\right) \neq 0 \Rightarrow \psi_{2}\left( \pm \frac{h}{2}\right) \neq 0 .
$$

However, Eq. (25) establishes

$$
\psi_{2}\left( \pm \frac{h}{2}\right)=C_{22}^{f} \bar{\psi}_{2}=0
$$

The above results demonstrate that the analytic formulation derived for the homogeneous nodes is not valid for heterogeneous node calculations. Because of the intranodal heterogeneity effects on the flux distribution and spectrum, the interface heterogeneous fluxes are not equal to the homogeneous ones available from the diffusion calculations, as was also shown by Koebke. ${ }^{3}$

To avoid the need of performing iteratively highorder heterogeneous calculations in order to treat all nonlinear effects, as is done in our COBAYA code, ${ }^{8}$ a new formulation is proposed. Here, the analytic relationships [Eq. (21)] are generalized to be applied to nonhomogeneous nodes.

For that, since $\bar{\psi}_{2}=0$ independently of matrix $\boldsymbol{C}^{\boldsymbol{f}}$, a kind of correction factor has to be included in order to preserve the average heterogeneous quantities from the homogeneous diffusion calculations. The analytic formulation provides the homogeneous fluxes across the interfaces, which are not the same as the heterogeneous ones calculated with a higher-level method. The flux from the homogeneous solution has to be discontinuous at the interfaces. Its ratio to the heterogeneous flux will define the so-called interface flux discontinuity (IFD) factors, or simply heterogeneity factors, because they take care only of errors due to the homogenization procedure.

The heterogeneous surface fluxes are then related to the homogenized ones by a $2 \times 2$ diagonal matrix of heterogeneity factors as follows:

$$
\boldsymbol{\phi}_{( \pm h / 2)}^{\text {hom }}=\left[\begin{array}{cc}
f_{f} & 0 \\
0 & f_{t h}
\end{array}\right] \boldsymbol{\phi}_{( \pm h / 2)}^{\text {het }} .
$$

Equation (26) expresses that there exists a scalar direct relationship per group between the heterogeneous and homogeneous fluxes across the interfaces. The heterogeneous, but not the homogeneous, interface fluxes must be continuous.

Then, for the single-assembly calculation,

$$
\boldsymbol{R} \boldsymbol{f} \phi_{( \pm h / 2)}^{h e t}=\boldsymbol{C}^{\boldsymbol{f}} \boldsymbol{R} \overline{\boldsymbol{\phi}} .
$$

And, in general,

$$
\boldsymbol{R} \boldsymbol{f} \boldsymbol{\phi}_{( \pm h / 2)}^{h e t}=\boldsymbol{C}^{\boldsymbol{f}} \boldsymbol{R} \overline{\boldsymbol{\phi}} \mp \frac{h}{2} \boldsymbol{C}^{\boldsymbol{j}} \boldsymbol{R} \boldsymbol{D}^{-1} \boldsymbol{J}_{( \pm h / 2)}-\boldsymbol{T}_{( \pm h / 2)} .
$$

This relation provides the theoretical basis for the generalized ACMFD formulation for multidimensional heterogeneous nodes by equaling the heterogeneous fluxes per group at both node sides of each interface, thus obtaining the net currents as a matrix function of nodeaverage fluxes. As internal heterogeneity vanishes, the heterogeneity IFD factors approach one, and in the limit of homogeneous nodes, such factors become the unity.

\section{SYNTHESIS OF INTRANODAL HETEROGENEITIES IN HETEROGENEITY INTERFACE FLUX DISCONTINUITY FACTORS}

The above-defined two-group heterogeneity IFD factors per nodal interface depend on the realistic neutronic and thermal-hydraulic properties inside the node as well as on its boundary conditions. Consequently, to compute accurate heterogeneity factors of application to 3-D nodal simulations of full pressurized water reactor (PWR) cores, previous to the 3-D simulation, 2-D detailed calculations of the whole core at different axial heights should be performed. Logically, the method is impractical as a tool for reactor analysis since the computed factors would change for each different cycle or loading pattern.

An alternative for computing accurate heterogeneity factors is to analyze their dependencies on the main heterogeneity variables from data independent of the loading pattern. For this, different 2-D lattice burnup singleassembly calculations per node type were developed. In addition, different representative cases of colorsets and whole core calculations were made. These lead to the separation of, in a complete and consistent way, the different effects on the heterogeneity factors. Making use of least squares techniques, the functional dependencies of each state variable are fitted.

Dependencies with some variables are linear. This is because the nonlinear contribution of the variables is small (local variables: water density and temperature, fuel temperature, boron and xenon plus samarium absorptions) or because it is treated in a time-explicit way 
(spectral history index). However, there are dependencies that are a function of the node boundary, in other words, a function of the changes in the nodal leakage, which modify the internal flux distribution. From this point of view, we are facing a nonlinear process because its own diffusion equation coefficients depend on the solution structure. Then, we have introduced additional variables, new boundary indexes, to account for the intragroup spectral effects due to changes in the boundary conditions. Table I summarizes the chosen feedback variables.

Once synthetized, the functional dependencies or derivatives, the 3-D nodal analysis can be carried out with enough accuracy. Using the node-averaged burnup as parametric interpolation variable, nodal factors will be calculated by correcting the nominal values with the actual local, history, and boundary conditions of the node in the actual core configuration, as follows:

$$
\begin{aligned}
\langle f\rangle^{\text {nodal }}= & \left\langle f_{0}\right\rangle+\sum_{l}\left\langle\frac{\partial f}{\partial v^{l}}\right\rangle\left\langle\Delta v^{l}\right\rangle \\
& +\left\langle\frac{\partial f}{\partial S H}\right\rangle\langle\Delta S H\rangle+\left\langle\frac{\partial f}{\partial B}\right\rangle\langle\Delta B\rangle,
\end{aligned}
$$

where the partial derivatives are included in the same nodal library as cross sections and basic neutron kinetic parameters and the changes in the state variables refer to the nominal values in the library. For each node type there is a library, which is independent of the core loading pattern.

\section{IV.A. Linear Effects (Local and Spectral History Effects)}

The heterogeneity IFD factor dependencies on the local and spectral history effects are analogous to that obtained for the nodal cross sections. As has been justified in Ref. 1, such dependencies have to be synthesized from 2-D lattice burnup single-assembly calculations, including nominal, branch, and off-nominal cases of coolant density and temperature, fuel temperature, and boron and xenon plus samarium absorptions. The variation ranges are chosen so that the core conditions to be analyzed are covered. The new nodal spectral history index, equivalencing the different burnup paths in parameter space, has been also formulated in Ref. 1 .

\section{TABLE I}

Local State and Generalized Variables

$$
\begin{array}{ll}
\text { Local variables }\left(v^{l}\right) \quad & \log \rho, \sqrt{T_{\text {mod }}}, \sqrt{T_{\text {fuel }}}, \\
& C_{\text {boron }}, \sum_{a b s 2}^{\mathrm{Xe}+\mathrm{Sm}}
\end{array}
$$

Generalized variables Spectral history index $(\mathrm{SH})$, boundary indexes $(B)$
In a single-assembly 2D calculation, eight heterogeneity factors will have to be computed for each quarterof-assembly node (one per group and interface). If, as usual, the node is diagonal-symmetric, only four factors per node need to be calculated (one factor per group and external or internal interface referred to its relative orientation to the assembly center).

Figure 1 indicates the dependence of the fast heterogeneity factor on coolant density. This factor corresponds to an external nodal interface of a standard $17 \times$ 17 fuel assembly, with $2.60 \mathrm{wt} \%$ enrichment and 16 wet annular burnable absorbers (waba's). Figure 1 shows that the factor can be easily modeled with a linear fit versus this local coolant density change. Reactivity errors due to the linear reduction of the heterogeneity factor dependencies on the local and spectral history variables lead one to conclude that second-order polynomial effects and cross terms can be neglected.

\section{IV.B. Nonlinear Effects (Neighborhood Effects) on Heterogeneity Interface Flux Discontinuity Factors}

The changes in the boundary conditions at each nodal interface or the existence of exposure-induced gradients within a node modify substantially the neutron spectrum and, then, the nodal heterogeneity IFD factors from those obtained by single-assembly calculations. The surroundings of a given node are modified when neighboring assemblies have different enrichment, control, or burnable absorber rods, peripheral gadolinium pins, or strong burnup gradients, or with the proximity of the reflector.

In all cases, the changes in the nodal leakage, represented by nonzero currents across the interassembly boundaries, modify the flux spectrum inside the assembly, and then, the ratio of the homogeneous surface-average flux to the hypothetical heterogeneous surface-average flux is modified. Therefore, the heterogeneity factors depend on the flux spectrum in a nonlinear way.

Let us suppose a mesh-fine homogeneous singleassembly calculation. Obviously, the flux distribution is

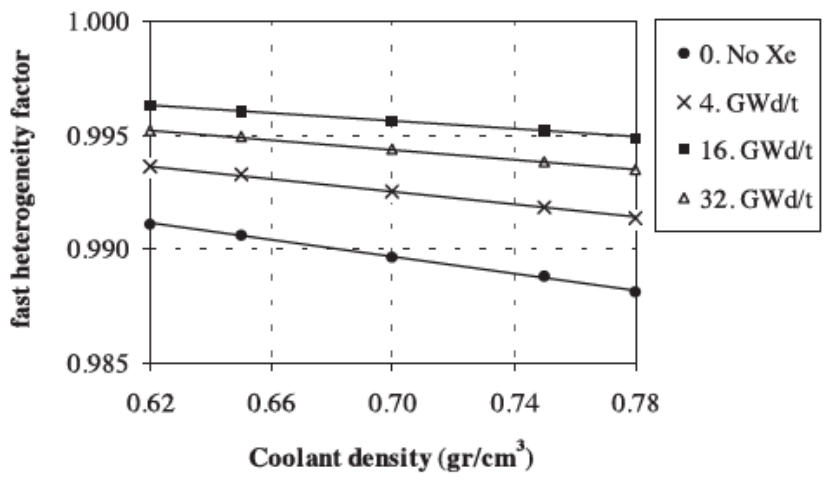

Fig. 1. Fast heterogeneity factor in an external nodal interface as function of coolant density. 
spatially flat, and then, the actual fluxes in the interfaces match the fluxes resulting from a coarse-mesh homogeneous single-assembly calculation. Therefore, the heterogeneity factors will be unity.

Let us suppose a mesh-fine heterogeneous singleassembly calculation. The flux distribution is not spatially flat because of the node internal structure; however, the flux resulting from a coarse-mesh homogeneous calculation would be flat (and then equal to the nodeaveraged flux). Then, the heterogeneity factors will be the ratios of heterogeneous to homogeneous surfaceaveraged fluxes, or ratios of the heterogeneous surfaceto-average flux in this case of zero net current boundary conditions.

In situations in which there is leakage across nodal interfaces, the flux structure is modified, and then heterogeneity factors will be poorly approximated by the surface-to-average flux ratio. Then, in order to isolate the effects due to the existence of nonzero leakage, additional calculations to the single-assembly have to be performed.

Let us notice node (i) in Fig. 2, initially taking part of an infinite lattice of identical assemblies. Suppose control is instantaneously introduced within the adjacent node $(i+1)$. In that moment there is internal flux redistribution. For the fast group, both the homogeneous and heterogeneous surface fluxes as well as the transverseintegrated heterogeneous flux distribution are plotted before (gray) and after (black) having introduced control within the node $(i+1)$. The thermal group shows similar behavior.

As can be seen, when the flux distribution changes, the homogeneous surface fluxes change at the same rate, so the ratio or heterogeneity factors $\phi_{\text {hom }}^{g} / \phi_{\text {het }}^{g}=f^{g}$ are independent of total leakage. Total leakage affects the homogenized flux through Eq. (21). However, it can be seen that the internal flux structure of the node under consideration [node (i)] has been modified with a higher effect in the region closer to the node-control interface. Hence, there is a directional response, which will affect the heterogeneity factors. For that reason the boundary variable must contain a directional component that takes into account information about the new internal flux structure directionality.

Thus, the neighborhood effect in the nodal correction factors has been related to the homogeneous flux gradients per group from those implicit in the singleassembly calculation [which are zero, as can be seen in Fig. 2 or in Eq. (30)]. This variable corrects in the first approximation the intranodal flux distribution induced by an instantaneous change of the boundary conditions.

If $\bar{\phi}$ represents the average flux in the node and $\phi_{\text {interface }}$ denotes the homogenized interface-averaged flux in each nodal interface, referred to as the relative orientation of each interface to the assembly center, the heterogeneity factor change due to the directionalinstantaneous neighborhood is proportional to

$$
\nabla \phi^{g}=\left(\frac{\phi_{\text {homogeneous interface }}^{g}-\bar{\phi}^{g}}{\bar{\phi}^{g}}\right) .
$$

In short, a separable correlation for the neighborhood effect in all nodal interface heterogeneity factors has been developed as a function of the instantaneous flux gradient per group. This parameterization is valid not only for the heterogeneity factors in the internal interfaces of the core but also for reflector-core interfaces.

The variation of the fast heterogenity factor for an external node interface from a standard $17 \times 17$ fuel assembly, with $2.60 \mathrm{wt} \%$ enrichment and 16 waba's, when modifying instantaneously its boundary conditions, is represented in Fig. 3. Five different representative cases of colorsets are developed: colorsets with $2.10 \mathrm{wt} \%, 0$ waba; $3.10 \mathrm{wt} \%$, 0 waba; $2.60 \mathrm{wt} \%$, 0 waba; $2.60 \mathrm{wt} \%, 24$ waba's; and $2.60 \mathrm{wt} \%$ with control, as well as a whole core calculation, where the node is in different peripheral positions. The factor variation is represented as a function of changes in both the fast and thermal directional variable. It can be seen how the functional dependence reduces to a linear correlation. In other words, the

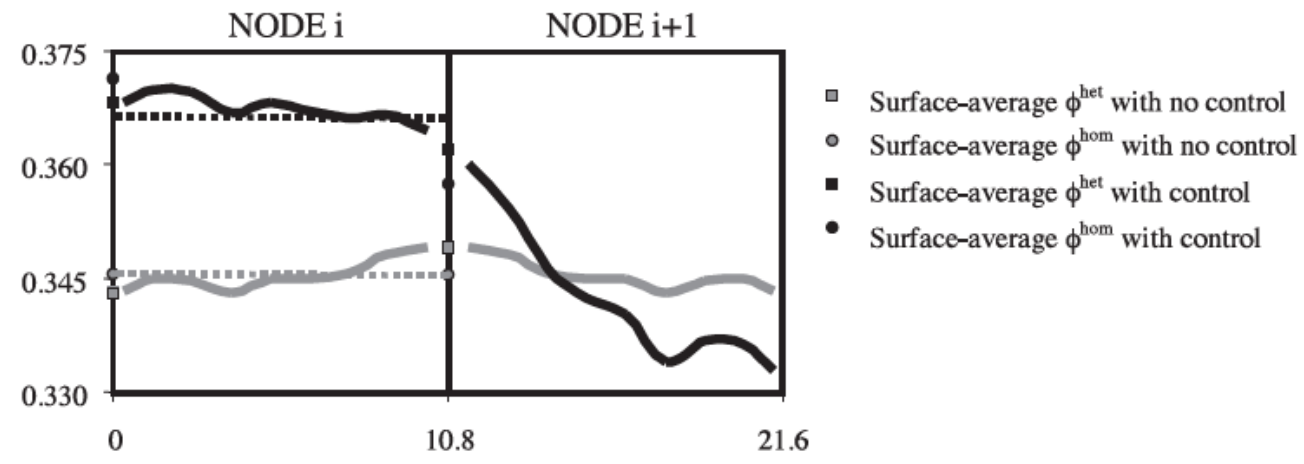

Fig. 2. Fast flux transverse-integrated distribution in two nodes from two equal adjacent assemblies, before (gray) and after (black) control rods have been inserted into node $(i+1)$. 

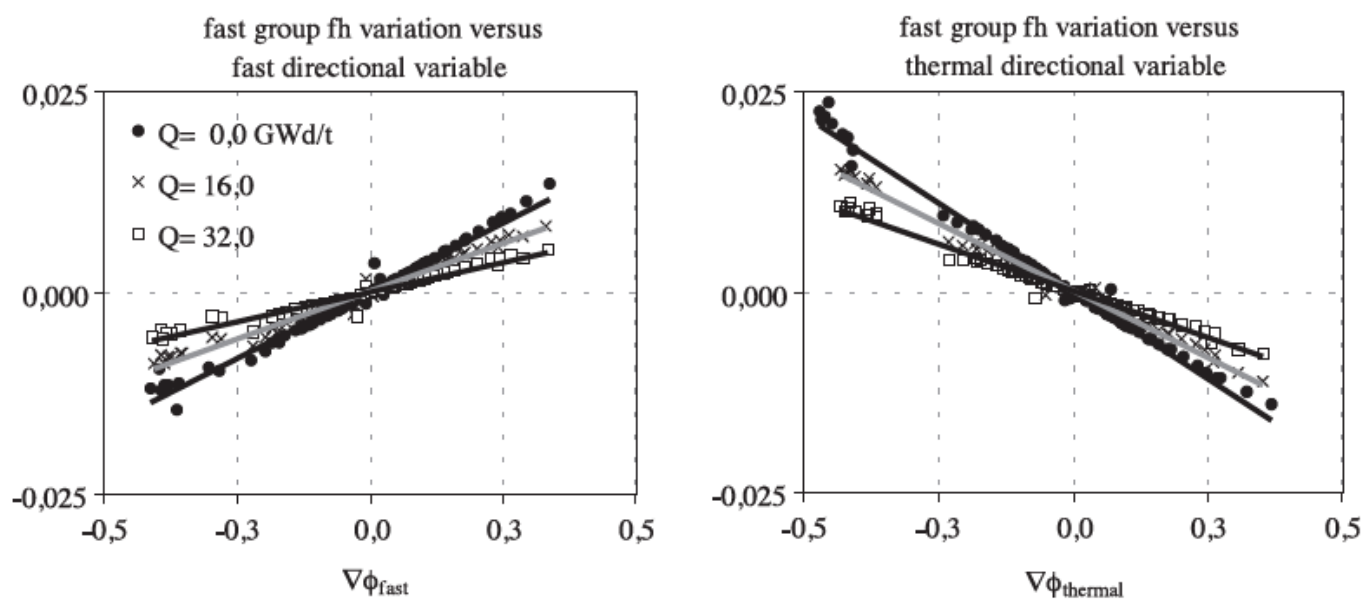

Fig. 3. Changes of the fast heterogeneity factor in an external nodal interface when modifying the nodal boundary conditions.

neighborhood effect in the correction factors is equivalent whatever the diverse surroundings of the node. The derivative changes significantly but smoothly with node burnup. The heterogeneity factor of the thermal group also shows similar behavior, so that factors in both groups depend on the flux gradients of the two groups.

It is then demonstrated that a single set of variables can be used for determining the neighborhood effects in heterogeneity factors due to all boundary conditions. The advantage of this approach is first that the task of computing needs to be performed only for a reduced number of conditions (as the two extreme colorsets), not necessarily for every different previous type, since it has been demonstrated that the funcional dependence is linear. Second, calculations have to be performed only once for each type of assembly, being the library independent of the cycle.

\section{IMPLEMENTATION}

The ACMFD formulation, generalized for heterogeneous nodes through heterogeneity IFD factors, determined by the above parameterization model, has been implemented in our two-group 2-D SIMULA2D code for steady-state calculations. This step, previous to the implantation of the methodology in the 3-D SIMULA3D and SIMTRAN (Ref. 12) codes, is necessary in order to carry out an exhaustive validation of the radial heterogeneity treatment.

SIMULA2D solves the two-group diffusion equation through a CMFD scheme. The code has been modified to include the developed formulation, based on the coupling equation (28). The problem is solved simultaneously for both neutron energy groups. The problem involves a linear system with a block pentadiagonal matrix in 2-D problems (block heptadiagonal in 3-D problems). Each block is a full $2 \times 2$ matrix, where coefficients represent the coupling of the average nodal flux of each node with their neighbors.

In order to accelerate the iteration in the fission source, the Wielandt eigenvalue shift method is used, following the solving method in PARCS (Ref. 13). Note that no local two-node problems are solved during the course of the CMFD iterations (as occurs in the PARCS method) since the nonlinear effects are considered explicitly in the nodal equivalent parameters - both the cross sections and heterogeneity IFD factors. These latter correct the interface net currents resulting from the CMFD approach.

The linear and nonlinear feedbacks on the nodal parameters have been shown to be a very effective way of accounting for the heterogeneity effects in two-group multidimensional coarse-mesh diffusion calculations. In other words, intranodal heterogeneity is being included in its own cross sections and heterogeneity factors, instead of being included in the nodal calculation process.

The current across the interface between two adjacent nodes $(i)(i+1)$ is related to the average fluxes in such nodes by

$$
\begin{aligned}
\boldsymbol{J}_{i \rightarrow i+1}= & -\frac{2}{h}\left[f_{i \rightarrow i+1}^{-1} A_{i}^{j}+\boldsymbol{f}_{i \rightarrow i+1}^{-1} A_{i+1}^{-1}\right]^{-1} \\
& \times\left[f_{i \rightarrow i+1}^{-1} A_{i \rightarrow i+1}^{f} \bar{\phi}_{i+1}-f_{i \rightarrow i+1}^{-1} A_{i \rightarrow i+1}^{f} \bar{\phi}_{i}\right],
\end{aligned}
$$

deduced from Eq. (28), where

where

$$
\begin{aligned}
\boldsymbol{A}^{j}=\boldsymbol{R}^{-1} \boldsymbol{C}^{j} \boldsymbol{R}= & \begin{array}{l}
\text { matrix depending } \\
\text { only on the node, } \\
\\
\text { not on the interface }
\end{array} \\
\boldsymbol{A}_{i \rightarrow i+1}^{f}=\boldsymbol{R}^{-1} \boldsymbol{C}^{f} \boldsymbol{R}-\boldsymbol{A}_{i \rightarrow i+1}^{\prime f}= & \begin{array}{l}
\text { matrix depending on } \\
\text { the nodal interface, }
\end{array}
\end{aligned}
$$




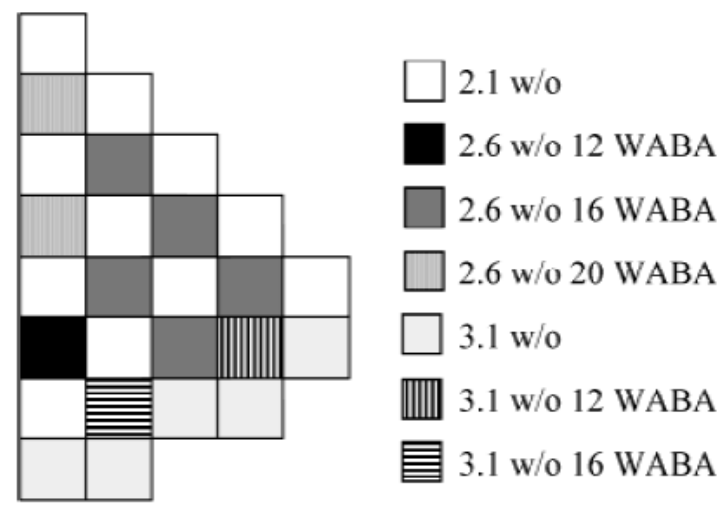

Fig. 4. Loading pattern in the first cycle of Ascó II NPP.

having expressed the transverse leakage term a function of the average flux in the following way:

$$
\boldsymbol{R}^{-1} \boldsymbol{T}_{i \rightarrow i+1}=\boldsymbol{A}_{i \rightarrow i+1}^{\prime f} \bar{\phi} .
$$

\section{VALIDATION}

The case under study is the initial core loading pattern of the Ascó II nuclear power plant (NPP), which is a Westinghouse-type PWR rated at $2775 \mathrm{MW}$ (thermal).
As can be seen in Fig. 4, this pattern contains seven different types of fuel assemblies, identified by the enrichment and the number of burnable poison pins. Each type of assembly represents a uranium oxide fuel assembly with different types of $17 \times 17$ pin cells. Six of these types are arranged in a checkerboard loading scheme, whereas the fuel assemblies of the highest reactivity are located at the core periphery. A steel shroud and a reflector zone surround the core.

Comparisons were made between the results obtained with the 2-D mesh-fine transport-corrected diffusion COBAYA code (reference code) and those obtained with the new 2-D SIMULA2D code. The nodal libraries to be used by SIMULA2D were generated for each type of fuel assembly and include all the local, history, and neighborhood derivatives.

The effects of intranodal gradients on the radial power distribution are shown in Fig. 5. First, Fig. 5a shows deviations at the beginning of the burnup cycle when both nodal cross sections and heterogeneity factors for each node are assumed to be those corresponding to the single-assembly calculations. These parameters can be easily reproduced from libraries, taking into account the local and spectral history feedbacks. At the beginning of the cycle and full power, the relative percent discrepancy is almost $2 \%$ and increases with the cycle burnup. When only the intranodal gradient effects are considered in the cross sections, the error decreases to $\sim 1 \%$ (Fig. $5 \mathrm{~b}$ ).

\begin{tabular}{|c|c|c|c|c|c|c|c|c|}
\hline \multicolumn{9}{|l|}{-0.24} \\
\hline-1.87 & -0.22 & & & & & & & \\
\hline |-1.78 & -0.22 & -0.21 & & & & & & \\
\hline-0.18 & -1.51 & -1.43 & -0.10 & & & & & \\
\hline-0.11 & -1.45 & -1.35 & -0.03 & 0.02 & & & & \\
\hline-1.55 & 0.02 & 0.02 & -1.20 & -1.11 & 0.31 & & & \\
\hline-1.42 & 0.13 & 0.11 & -1.07 & -0.97 & 0.38 & 0.45 & & \\
\hline 0.25 & -1.11 & -1.00 & 0.39 & 0.44 & -0.77 & -0.56 & 0.99 & \\
\hline 0.32 & -0.90 & -0.81 & 0.52 & 0.59 & -0.02 & 0.34 & 1.06 & 0.93 \\
\hline-0.47 & 0.58 & 0.62 & -0.62 & 0.06 & 0.06 & 0.46 & 0.89 & 0.52 \\
\hline-0.48 & 0.83 & 0.92 & 0.18 & 0.93 & 0.71 & 1.14 & 0.89 & -0.02 \\
\hline 0.89 & -0.87 & -0.19 & 0.96 & 0.98 & 1.31 & 1.00 & & \\
\hline 1.11 & 0.07 & 0.93 & 1.05 & 0.36 & 0.54 & 0.21 & & \\
\hline 0.75 & 1.24 & 1.27 & & & & & & \\
\hline 0.41 & 0.46 & 0.23 & & & & & & \\
\hline
\end{tabular}

(a)

\begin{tabular}{|c|c|c|c|c|c|c|}
\hline 0.14 & & & & & & \\
\hline 0.76 & 0.08 & & & & & \\
\hline 0.78 & -0.01 & -0.08 & & & & \\
\hline-0.03 & 0.67 & 0.68 & -0.17 & & & \\
\hline :-0.07 & 0.62 & 0.64 & $\begin{array}{ll}-0.21 & -0.27\end{array}$ & & & \\
\hline 0.65 & -0.12 & -0.21 & $\begin{array}{ll}0.57 & 0.53\end{array}$ & -0.29 & & \\
\hline 0.60 & -0.18 & -0.28 & $\begin{array}{ll}0.52 & 0.47\end{array}$ & $-0.38-$ & -0.50 & \\
\hline-0.23 & 0.48 & 0.50 & $-0.32 \quad-0.40$ & 0.34 & 0.28 & -0.46 \\
\hline-0.32 & 0.45 & 0.43 & $-0.42-0.61$ & 0.15 & 0.14 & $-0.79-0.98$ \\
\hline 0.35 & -0.37 & -0.46 & $\begin{array}{|ll|}0.23 & 0.06 \\
\end{array}$ & 0.36 & 0.19 & $0.43-0.23$ \\
\hline 0.32 & -0.59 & -0.72 & $-0.03 \quad-0.12$ & 0.16 & 0.09 & $-0.48 \quad-0.89$ \\
\hline-0.63 & 0.59 & 0.40 & $\begin{array}{|ll|}0.38 & 0.03 \\
\end{array}$ & 0.09 & -0.46 & \\
\hline I. 1.06 & 0.39 & 0.28 & $-0.38 \quad-0.82$ & $-0.54-$ & -0.68 & \\
\hline 0.33 & 0.24 & -0.04 & & & & \\
\hline-0.60 & -0.52 & -0.58 & & & & \\
\hline
\end{tabular}

(b)

Fig. 5. Errors (\%) in power distributions to $0.0 \mathrm{GWd} / \mathrm{t}$, hot full power, all rods out, with (a) cross sections and heterogeneity factors from assembly calculations and (b) with intranodal flux gradient effect corrections only in cross sections. 


\begin{tabular}{|c|c|c|c|c|c|c|c|}
\hline 0.77 & & & & & & & \\
\hline-0.07 & 0.43 & & & & & & \\
\hline-0.05 & 0.30 & 0.21 & & & & & \\
\hline 0.43 & 0.15 & 0.12 & 0.13 & & & & \\
\hline 0.39 & 0.12 & 0.11 & 0.09 & 0.07 & & & \\
\hline-0.14 & 0.21 & 0.10 & 0.07 & 0.02 & 0.06 & & \\
\hline-0.17 & 0.18 & 0.08 & 0.04 & 0.02 & 0.03 & 0.02 & \\
\hline 0.33 & 0.07 & 0.07 & 0.05 & 0.04 & -0.03 & -0.03 & 0.17 \\
\hline 0.27 & 0.10 & 0.06 & 0.03 & -0.03 & -0.05 & 0.08 & $\begin{array}{ll}0.34 & 0.75 \\
\end{array}$ \\
\hline-0.28 & 0.21 & 0.10 & -0.08 & -0.15 & 0.34 & 0.17 & $-0.37-0.53$ \\
\hline-0.20 & 0.36 & 0.32 & 0.04 & 0.01 & 0.22 & 0.32 & $-0.38 \quad-0.46$ \\
\hline 0.34 & -0.46 & -0.50 & -0.22 & -0.19 & -0.26 & -0.32 & \\
\hline 0.45 & -0.19 & 0.12 & -0.31 & -0.35 & -0.34 & -0.22 & \\
\hline-0.64 & -0.52 & -0.21 & & & & & \\
\hline-0.38 & -0.45 & -0.26 & & & & & \\
\hline
\end{tabular}

(a)

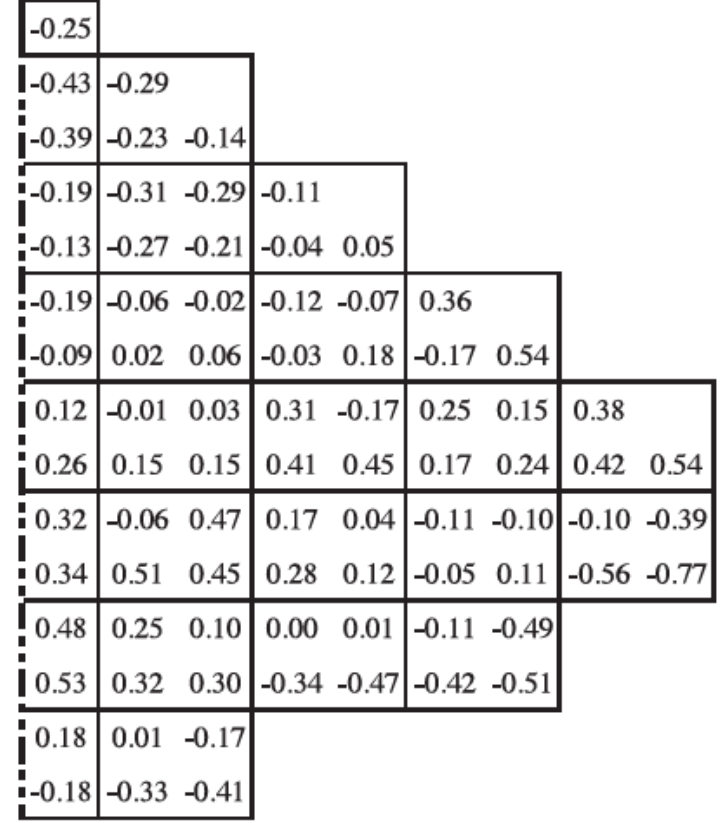

(b)

Fig. 6. Errors (\%) in power distributions at (a) $0.0 \mathrm{GWd} / \mathrm{t}$, hot full power, all rods out and at (b) $12.0 \mathrm{GWd} / \mathrm{t}$ with intranodal gradient corrections also in heterogeneity factors.

When the intranodal gradient effects are included (through directional neighborhood variables) also in the heterogeneity factors, the obtained results are summarized in Fig. 6 at different cycle burnups. The results indicate that the power distributions predicted by the SIMULA2D method over the whole core are very accurate when the intranodal effects are considerated on all the nodal parameters. Errors never overpassed $0.77 \%$ for all nodes and all cycle burnups. Note that the assemblies facing the core baffle with two interfaces do not have the higher errors.

Table II compares the root-mean-square error in power along the cycle burnup, as well as the error in maximum powers per assembly. We note that there is good agreement with the reference case, from the beginning until the end of the cycle.

A comparison of the computing times for the various solutions is presented in Table III, where we note that the SIMULA2D solutions required approximately half the computing time of the previous SIMULA2D nodal method [including the older formulation Eq. (2)]. On the other hand, the new nodal code reduces the computing time of the reference solution by a factor of 40 for usual 2-D problems. The convergency looks quite satisfactory, as can be seen in Fig. 7.

\section{CONCLUSIONS}

In this paper, a new nonlinear treatment of the heterogeneity effects on nodal interface two-group heterogeneity factors was developed and tested on a real reactor problem. These factors complete the set of equivalent parameters that allow predicting of the heterogeneous solutions from few-group diffusion CMFD equations.

The use of these heterogeneity IFD factors at every interface (one per energy group) leads to a generalization of the ACMFD methodology for heterogeneous nodes. The major advantage of this formulation is that it

TABLE II

Comparison of Nodal Solutions with Reference Calculations

\begin{tabular}{|l|c|l|l|l|l|l|l|}
\hline Cycle burnup (GWd/t), hot full power & 0 & 0.150 & 2 & 4 & 8 & 12 & 14.8 \\
Root-mean-square percent error in power & 0.26 & 0.35 & 0.27 & 0.26 & 0.23 & 0.29 & 0.34 \\
Percent error in maximum power & -0.08 & 0.41 & -0.24 & 0.08 & 0.08 & 0.25 & 0.02 \\
\hline
\end{tabular}


TABLE III

Accumulated Computing Times on HP-780 for the Various Solutions*

\begin{tabular}{|l|c|c|c|}
\hline $\begin{array}{c}\text { Burnup } \\
(\mathrm{GWd} / \mathrm{t})\end{array}$ & $\begin{array}{c}\text { Reference } \\
\text { Code }\end{array}$ & $\begin{array}{c}\text { Previous } \\
\text { SIMULA2D }\end{array}$ & $\begin{array}{c}\text { New } \\
\text { SIMULA2D }\end{array}$ \\
\hline 0 & 269 & 7 & 5 \\
0.150 & 398 & 12 & 7 \\
2 & 781 & 24 & 12 \\
4 & 1019 & 32 & 16 \\
8 & 1261 & 39 & 20 \\
12 & 1517 & 50 & 23 \\
14.8 & 1648 & 55 & 25 \\
\hline
\end{tabular}

*The accumulated computing times are in seconds.

does not need to be used iteratively with a higher-level nodal method. This is because first, it includes by itself the spectrum and coarse-mesh effects, making use of matrix relationships involving nodal eigenvalues and eigenvectors, and second, nonlinear effects are considered explicitly in the cross section and heterogeneity factor feedbacks.

The numerical results show that the converged solutions are fast and accurate. Comparisons have shown quite satisfactory results for most heterogeneities, even if some additional work is needed for very difficult configurations.

In the future, extension to a 3-D production code is envisaged. It will provide a tool for performing faster 3-D nodal calculations, which is very suitable for transient analysis or on-line applications in NPPs. The formulation can be incorporated into any CMFD code in a straightforward manner.

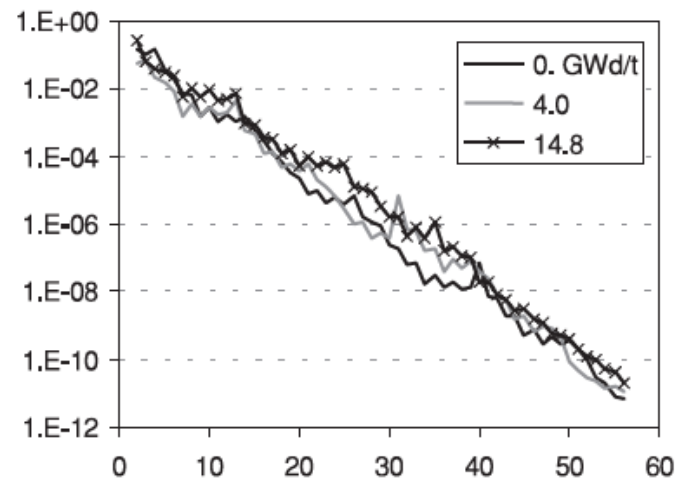

Fig. 7. Convergence ratio: maximum nodal deviation versus iteration number (double precision), at the beginning, middle, and end of the cycle.

\section{REFERENCES}

1. N. GARCÍA-HERRANZ, J. M. ARAGONÉS, O. CABELLOS, and C. AHNERT, "Dependence of the Nodal Homogenized Two-Group Cross Sections on Intranodal Flux-Spectrum, Burnup and History," Proc. Mathematics and Computation, Reactor Physics and Environmental Analysis in Nuclear Applications, Madrid, Spain, September 27-30, 1999, Vol. 1 (1999).

2. Y. A. CHAO, "Coarse Mesh Finite Difference Methods and Applications," Proc. Int. Topl. Mtg. Advances in Reactor Physics and Mathematics and Computation into the Next Millennium (PHYSOR 2000), Pittsburgh, Pennsylvania, May 7-12, 2000, American Nuclear Society (2000) (CD-ROM).

3. K. KOEBKE, "Advances in Homogenization and Dehomogenization," Proc. Int. Topl. Mtg. Advances in Mathematical Methods for the Solution of Nuclear Engineering Problems, Munich, Germany, Vol. 2, p. 59, Fachinformationszentrum Energie, Physik, Mathematik (1981).

4. K. S. SMITH, "Assembly Homogenization Techniques for Light Water Reactor Analysis," Prog. Nucl. Energy, 17, 303 (1986)

5. K. S. SMITH, "Nodal Method Storage Reduction by Nonlinear Iteration," Trans. Am. Nucl. Soc., 44, 265 (1983).

6. M. R. WAGNER, K. KOEBKE, and H. J. WINTER, "A Nonlinear Extension of the Nodal Expansion Method," Proc. Int. Topl. Mtg. Advances in Mathematical Methods for the Solution of Nuclear Engineering Problems, Munich, Germany, Vol. 2, p. 43, Fachinformationszentrum Energie, Physik, Mathematik (1981).

7. C. AHNERT and J. M. ARAGONÉS, "A Coupled Fine/ Coarse-Mesh Few-Group Diffusion Method," Trans. Am. Nucl. Soc., 47, 414 (1984).

8. J. M. ARAGONÉS and C. AHNERT, "A Linear Discontinuous Finite Difference Formulation for Synthetic CoarseMesh Few-Group Diffusion Calculations," Nucl. Sci. Eng, 94, 309 (1986).

9. O. CABELlOS, J. M. ARAGONÉS, and C. AHNERT, "Generalized Effects in Two Group Cross Sections and Discontinuity Factors in the DELFOS Code for PWR Cores," Proc. Mathematics and Computation, Reactor Physics and Environmental Analysis in Nuclear Applications, Madrid, Spain, September 27-30, 1999, Vol. 1, p. 117 (1999).

10. N. GARCÍA-HERRANZ, "Desarrollo de un Modelo Nodal 3D, con Efectos de Heterogeneidad en las Secciones Eficaces y Factores de Discontinuidad, para Análisis de Núcleos de Agua a Presión," Doctoral Thesis, Escuela Técnica Superior de Ingenieros Industriales (2000). 
11. Y. A. CHAO, "A Theoretical Analysis of the Coarse Mesh Finite Difference Representation in Advanced Nodal Methods," Proc. Mathematics and Computation, Reactor Physics and Environmental Analysis in Nuclear Applications, Madrid, Spain, September 27-30, 1999, Vol. 1, p. 117 (1999).

12. J. M. ARAGONÉS, C. AHNERT, and O. CABELLOS, "Methods and Performance at the Three-Dimensional Pressur- ized Water Reactor Core Dynamics SIMTRAN On-Line Code," Nucl. Sci. Eng., 124, 111 (1996).

13. H. G. JOO, D. A. BARBER, G. JIANG, and T. J. DOWNAR, "PARCS, A Multi-Dimensional Two-Group Reactor Kinetics Code Based on the Nonlineal Analytic Nodal Method," PARCS/NRC-V1.00. 